\title{
Erratum to: TERT Promoter Mutation and Telomere Length in Salivary Gland Tumors
}

\author{
Heejin Kim ${ }^{1,2}$ • Dongbin Ahn ${ }^{1}$. Jin Ho Sohn ${ }^{1}$ - Yong-Hee Kim ${ }^{3}$. Jae-Ho Lee ${ }^{4}$. \\ Hyunsu Lee ${ }^{4}$ (D)
}

Published online: 4 September 2017

(C) Arányi Lajos Foundation 2017

\section{Erratum to: Pathol Oncol Res https://doi.org/10.1007/s12253-017-0275-6}

The original version of this article unfortunately contained an error in Fig. 1 and omitted acknowledgment section.

The online version of the original article can be found at https://doi.org/ 10.1007/s12253-017-0275-6

\section{Hyunsu Lee}

neuroana@dsmc.or.kr

Heejin Kim

heejin0520@daum.net

Dongbin Ahn

godlikeu@naver.com

Jin Ho Sohn

entgodlikeu@gmail.com

Yong-Hee Kim

tolerance@knu.ac.kr

Jae-Ho Lee

anato82@dsmc.or.kr

1 Department of Otolaryngology-Head and Neck Surgery, Kyungpook National University, Daegu, Republic of Korea

2 Department of Otorhinolaryngology-Head and Neck Surgery, Hallym University, Dongtan Sacred Heart Hospital,

Hwaseong, Republic of Korea

3 Department of Microbiology, Kyungpook National University School of Medicine, Daegu, Republic of Korea

4 Department of Anatomy, Keimyung University School of Medicine, 1095, Dalgubeol-daero, Dalseo-gu, Daegu, Republic of Korea
In Fig. 1, b and c box plots were erroneously inserted. The corrected Fig. 1 is shown below.

The omitted acknowledgment section is given below.

This research was supported by the Keimyung University Research Grant of 2016.

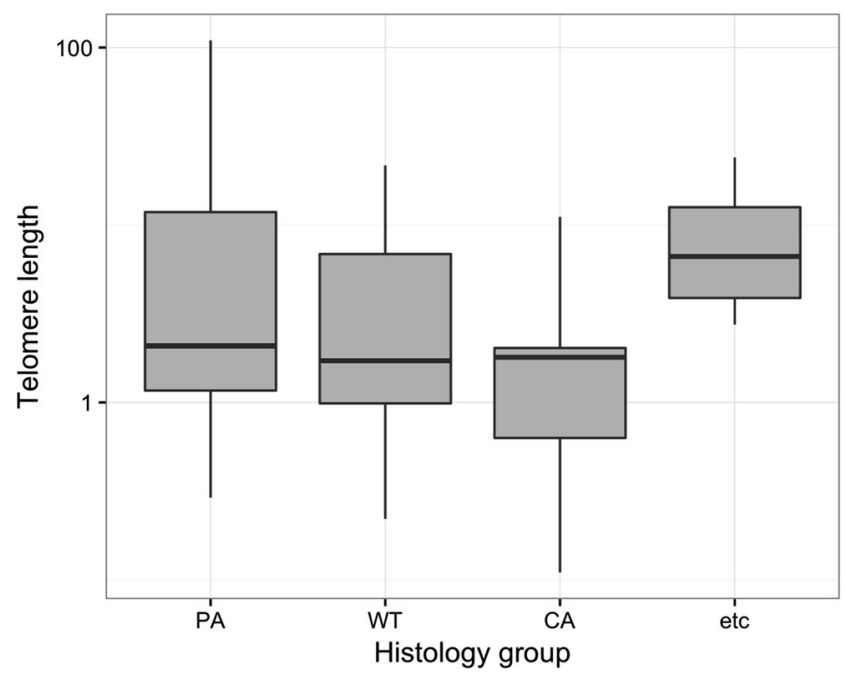

Fig. 1 Box plot of the telomere length for each histology group. The vertical line extends from the smallest non-outlier to the largest nonoutlier. The box was drawn from 25 to 75 percentiles. The thick horizontal line in the box means the median of the data. PA, pleomorphic adenoma; WT, Warthin tumor; CA, carcinoma; etc., another benign tumor 